\title{
Relaxation Processes in a Condensed Bose Gas*
}

\author{
Ulrich Eckern \\ Institut für Theorie der Kondensierten Materie, Universität Karlsruhe, Karlsruhe, \\ Federal Republic of Germany
}

We develop, on the basis of the self-consistent mean-field approximation, the kinetic theory for a dilute Bose gas below the Bose-Einstein transition temperature. The collision operator in the Boltzmann equation is calculated by golden rule arguments, and the momentum and temperature dependences of the scattering rates are determined. As an application, we consider the relaxation of a nonequilibrium distribution of the quasiparticles. We discuss the relevance of our calculation for the (hypothetical) condensed state of spinpolarized hydrogen.

\section{INTRODUCTION}

Since the recent experimental stabilization of spin-polarized hydrogen, ${ }^{1}$ theoretical attention has focused on the bosonlike nature of such a fluid at low temperatures and low densities. ${ }^{2-4}$ Should it be possible to stabilize about $10^{19}$ atoms $/ \mathrm{cm}^{3}$, one expects the system to undergo a Bose-Einstein transition at a temperature of about $70 \mathrm{mK}$. In a large magnetic field, say of order $10 \mathrm{~T}$, atomic hydrogen will behave in many ways like a twocomponent spin-1/2 Bose fluid, the two states being the two lowest hyperfine states. Consequently, attention has been devoted mainly to the magnetic properties. For example, if both states exhibit long-range order, one expects the perpendicular magnetization to acquire a spontaneous value. However, the stability of such a state is a delicate question. ${ }^{4}{ }^{\dagger}$

In the construction of hydrodynamic equations for the condensed system, the question of the microscopic time scales arises: these determine the limit of any hydrodynamic theory, while on the other hand, valuable information about the microscopic nature of the state under consideration can be obtained by comparing experimental and theoretical results for the parameters in the hydrodynamic equations. In this article, we want to study, *Zur Erlangung der Lehrbefähigung für das Lehrgebiet Physik der Fakultät für Physik der Universität Karlsruhe vorgelegte Habilitationsschrift. tFor a review of spin-polarized hydrogen, see Ref. 5 . 
as an important example, the relaxation rate of a nonequilibrium distribution of the quasiparticles. For simplicity, we consider a one-component Bose system.

The microscopic theory of the phase transition in a dilute Bose fluid has been investigated by many authors, for example, Bogoliubov, ${ }^{6}$ Belyaev ${ }^{7}$ Martin, ${ }^{8}$ and Hohenberg and Martin. ${ }^{9}$ As in the ideal gas transition, a special role has to be assigned to the zero-momentum occupation number, which has a macroscopic value below the transition temperature $T_{c}$. The quantity $\psi(\mathbf{r})=\langle\bar{\psi}(\mathbf{r})\rangle$, where $\bar{\psi}(\mathbf{r})$ is the usual annihilation operator and the brackets denote the thermodynamic average, appears as a kind of external field in the equations of motion; $n_{0}=|\psi|^{2}$ is the number density of condensed atoms. Basically, two approximation schemes have been developed: the gapless vs. the conserving approximation. In the equilibrium calculations of Bogoliubov ${ }^{6}$ and Belyaev ${ }^{7}$ it is guaranteed that in the considered order in perturbation theory the single-particle excitation spectrum is gapless (which is a theorem due to Hugenholtz and Pines ${ }^{10}$ ). Note also the more recent investigations, ${ }^{11,12}$ which identify and resolve certain difficulties of the perturbation expansion connected with the long-wavelength behavior of the spectrum. In a nonequilibrium calculation, on the other hand, it is preferable to use the self-consistent (and thus conserving) scheme in the sense as described by Hohenberg and Martin. ${ }^{*}$ In this approach, the singleparticle spectrum has a gap ${ }^{13}$ the magnitude of which is, however, always smaller than the order to which the calculation is valid. We use this approach in lowest order (mean-field approximation) to construct the quasiparticles of the kinetic theory.

The concept of kinetic theories/kinetic equations has been discussed by many authors, but especially with applications to superfluid helium by Khalatnikov. ${ }^{14}$ The kinetic equation (or Boltzmann equation) can be derived from the equations of motion for the Green's function, as outlined, for example, very clearly by Aronov and Gurevich ${ }^{15}$ for superconductors. The resulting equations apply to situations in which the space and time dependence of the various physical quantities is sufficiently slow, and provided the quasiparticles are well defined. To be more concrete, consider a Bose gas interacting via a repulsive pointlike potential $V(\mathbf{r})=v \delta(\mathbf{r})$. It should be noted that an expansion with respect to the potential has a very limited range of applicability; however, certain summations can be performed, ${ }^{7}$ which essentially replace $v$ by the scattering length $a$, through $\dagger$

$$
v-v^{2} \sum_{p} \frac{m}{p^{2}}+\cdots=\frac{4 \pi \hbar^{2} a}{m}
$$

${ }^{*}$ Ref. 9 contains a detailed discussion of the two approaches.

tSee also, for example, the discussion in Ref. 16, Chapters 1 and 5. 
where $m$ is the mass. The expansion parameter then turns out to be $a / l$ where $l$ is the average distance between particles ( $\rho=l^{-3}$ is the total density). Although we often keep $v$ to simplify some notations, Eq. (1) should be understood. In the Bogoliubov approximation, the single-particle spectrum has the form

$$
E_{p}^{\mathrm{B}}=\left(\xi_{p}^{2}+2 \xi_{p} n_{0} \nu\right)^{1 / 2}, \quad \xi_{p}=p^{2} / 2 m
$$

which may be considered temperature dependent through $n_{0}$. We then estimate the kinetic theory to be applicable provided*

$$
\hbar \omega \ll n_{0} v ; \quad \hbar|\mathbf{q}| \ll\left(m n_{0} v\right)^{1 / 2}
$$

where $\omega$ and $q$ are the characteristic frequency and wave vector, respectively. Assuming that the critical temperature is given by the ideal gas formula,

$$
k_{\mathrm{B}} T_{c} \approx 3.3 \hbar^{2} / m l^{2}
$$

we can write Eq. (3) in the form

$$
\hbar \omega \ll \frac{a}{l} \frac{n_{0}}{\rho} k_{\mathrm{B}} T_{c} ; \quad|\mathbf{q}| \ll\left(\frac{a}{l} \frac{n_{0}}{\rho}\right)^{1 / 2} l^{-1}
$$

The requirement of well-defined quasiparticles leads to the condition $\hbar / \tau \ll$ $n_{0} v$, where $\tau$ is a scattering time. Anticipating some of the following results, we find from this condition

$$
\varepsilon=\frac{T_{c}-T}{T_{c}} \gg \frac{a}{l}
$$

in agreement with Ref. 17. Thus we have to exclude a small temperature region near $T_{c}$, and this turns out to be just the critical region as given by the Ginzburg criterion, ${ }^{18}$

$$
\varepsilon_{\text {crit }} \approx k_{\mathrm{B}}^{2} /\left[\xi(0)^{3} \Delta C\right]^{2}
$$

where we estimate the coherence length $\xi(0)$ from the Gross-Pitaevski ${ }^{19}$ equation, $\xi(0) \approx l(l / a)^{1 / 2}$, and the specific heat jump by $\Delta C \approx k_{\mathrm{B}} \rho a / l$. We keep in mind applications to spin-polarized hydrogen, for which $a / l \approx 10^{-2}$ for the discussed density. ${ }^{20}$

The remaining sections are organized as follows. In Section 2, we outline the kinetic equations. Section 3 contains a discussion of the collision operators, and we calculate the temperature and momentum dependence of the scattering rates. The relaxation rate of a nonequilibrium distribution in a spatially homogeneous situation is determined in Section 4, and Section

* Note, however, that the kinetic theory in addition ignores certain effects that happen on a scale given by the gap in the spectrum (see Section 5 and Appendix B). 
5 contains some concluding remarks. Appendix A demonstrates the derivation of the scattering rate with the Green's function technique, and Appendix $B$ contains a discussion of the mean-field equations in the collisionless regime. Some of the results were reported earlier. ${ }^{21}$

\section{THE KINETIC EQUATIONS}

For illustration, we repeat a few well-known microscopic considerations. Consider the Hamiltonian of a system of bosons in an external potential $U_{x}$, interacting via a repulsive point interaction $v$ :

$$
\begin{aligned}
& \mathscr{H}=\int d^{3} r \bar{h} \\
& \bar{h}=\frac{\hbar^{2}}{2 m}(\nabla \bar{\psi})^{+}(\nabla \bar{\psi})+\bar{\psi}^{+} U_{x} \bar{\psi}+\frac{v}{2} \bar{\psi}^{+} \bar{\psi}^{+} \bar{\psi} \bar{\psi}
\end{aligned}
$$

where $\bar{\psi}(\mathbf{r})$ and $\bar{\psi}^{+}(\mathbf{r})$ are the usual field operators. In the first step, we perform a gauge transformation,

$$
\bar{\psi}=\hat{\psi} e^{i \chi}, \quad \bar{\psi}^{+}=\hat{\psi}^{+} e^{-i x}
$$

where $\chi$ is in general space and time dependent. We define

$$
\mathbf{v}_{s}=\frac{\hbar}{m} \nabla \chi ; \quad \mu_{c}=-\hbar \dot{\chi}
$$

and obtain the new Hamiltonian $\hat{h}$ (only the term quadratic in the field operators is modified):

$$
\hat{h}=\frac{\hbar^{2}}{2 m}\left[\left(\nabla+i \frac{m \mathbf{v}_{s}}{\hbar}\right) \hat{\psi}\right]^{+}\left[\left(\nabla+i \frac{m \mathbf{v}_{s}}{\hbar}\right) \hat{\psi}\right]+\hat{\psi}^{+}\left(U_{x}-\mu_{c}\right) \hat{\psi}+\cdots
$$

As a direct consequence of the definition in (8), we find $m \dot{\mathbf{v}}_{s}=-\nabla \mu_{c}$, and

$$
m\left[\dot{\mathbf{v}}_{s}+\left(\mathbf{v}_{s} \cdot \nabla\right) \mathbf{v}_{s}\right]=-\nabla \phi+\mathbf{F}_{x}
$$

where $\mathbf{F}_{x}=-\nabla U_{x}$ is the external force, and we introduced

$$
\phi=\mu_{c}-\frac{m}{2} v_{s}^{2}-U_{x}
$$

Also, the current operator transforms to

$$
\mathbf{j}=\frac{i \hbar}{2 m}\left(\hat{\psi} \nabla \hat{\psi}^{+}-\hat{\psi}^{+} \nabla \hat{\psi}\right)+\mathbf{v}_{s} \hat{\psi}^{+} \hat{\psi}
$$

In the second step, we take care of the special role of the condensate by 
introducing

$$
\hat{\psi}=\langle\hat{\psi}\rangle+\hat{\varphi}
$$

and we choose the phase in such a way that $\langle\hat{\psi}\rangle$ is a real quantity. Then it is clear that $\mathbf{V}_{s}$ becomes just the superfluid velocity, and $\phi$ may be called the chemical potential of the condensate (we do not want to discuss whether the $\frac{1}{2} m v_{s}^{2}$ should be included within such a notation). Clearly, we may write $\langle\hat{\psi}\rangle=\sqrt{n_{0}}$, where $n_{0}$ is the density of condensed particles. We now insert (13) into (9), treat the interaction in mean field approximation, and neglect small gradient terms. Then it is straightforward to derive the expression for the local energy density $e$.

The result is $*$

$$
\begin{aligned}
e= & -n_{0} \phi+\frac{1}{2} v n_{0}^{2}-\frac{1}{4 v}\left(\sigma_{11}-2 n_{0} v\right)^{2}-\frac{1}{2 v}\left(\sigma_{12}-n_{0} v\right)^{2} \\
& +\frac{1}{2} \operatorname{tr} \sum_{p}\left\{\left[\begin{array}{cc}
\varepsilon_{p}+\mathbf{p} \cdot \mathbf{v}_{s} & \sigma_{12} \\
\sigma_{12} & \varepsilon_{p}-\mathbf{p} \cdot \mathbf{v}_{s}
\end{array}\right] \hat{n}(\mathbf{p}, \mathbf{r}, t)\right\}
\end{aligned}
$$

Here, we defined the diagonal part of the self-energy $\sigma_{11}=2 v \rho$, where the total density $\rho$ is the sum of the particles in the condensate and the excited particles,

$$
\rho=n_{0}+\sum_{p} \hat{n}_{11}(\mathbf{p}, \mathbf{r}, t)
$$

and the off-diagonal self-energy $\sigma_{12}$ is given by

$$
\sigma_{12} / v=n_{0}+\sum_{p} \hat{n}_{12}(\mathbf{p}, \mathbf{r}, t)
$$

Also, we introduced the center-of-mass coordinate $\mathbf{r}$ and the Fourier transform of the relative coordinate $\mathbf{p} ; \hat{n}(\mathbf{p}, \mathbf{r}, t)$ is directly related to the equaltime (" $<$ ") Green's function of the excitations, expressed in these variables ( $\hat{n}$ is a $2 \times 2$ matrix). Finally,

$$
\varepsilon_{p}=\xi_{p}+\sigma_{11}-\phi
$$

Clearly, Eq. (14) can also be derived with the help of the Green's function technique.

Now it becomes evident how to proceed. In the usual way, we perform a Bogoliubov transformation, and introduce the amplitudes $u_{p}, v_{p}$ given by

$$
u_{p}^{2}=\frac{1}{2}\left(\varepsilon_{p} / E_{p}+1\right), \quad v_{p}^{2}=\frac{1}{2}\left(\varepsilon_{p} / E_{p}-1\right)
$$

*We omit the inverse of the volume in front of every momentum summation. 
with $u_{p}^{2}-v_{p}^{2}=1$. The last term in (14) then takes the simple form

$$
\sum_{p} \check{E}_{p}\left(n_{p}-v_{p}^{2}\right)
$$

where $n_{p}=n(\mathbf{p}, \mathbf{r}, t)$ is the scalar distribution function of the excitations. The spectrum is obtained as

$$
\check{E}_{p}=E_{p}+\mathbf{p} \cdot \mathbf{v}_{s}, \quad E_{p}=\left(\varepsilon_{p}^{2}-\sigma_{12}^{2}\right)^{1 / 2}
$$

which can also be written in the form

$$
E_{p}=\left(\Delta^{2}+2 m \xi_{p} c^{2}+\xi_{p}^{2}\right)^{1 / 2}
$$

with $\Delta^{2}=\left(\sigma_{11}-\phi\right)^{2}-\sigma_{12}^{2}, m c^{2}=\sigma_{11}-\phi$. Furthermore, particle and current density are given by

$$
\begin{gathered}
\rho=n_{0}+\sum_{p}\left[u_{p}^{2} n_{p}+v_{p}^{2}\left(1+n_{p}\right)\right] \\
\mathbf{j}=\rho \mathbf{v}_{s}+\mathbf{j}_{1}, \quad \mathbf{j}_{1}=\sum_{p} \frac{\mathbf{p}}{m} n_{p}
\end{gathered}
$$

Finally, the distribution function of the excitations $n_{p}$ has to be calculated from the Boltzmann equation:

$$
\dot{n}_{p}+\frac{\partial \check{E}_{p}}{\partial \mathbf{p}} \frac{\partial n_{p}}{\partial \mathbf{r}}-\frac{\partial \check{E}_{p}}{\partial \mathbf{r}} \frac{\partial n_{p}}{\partial \mathbf{p}}=I\left\{n_{p}\right\}
$$

where the collision operator $I$ describes the change in time of $n_{p}$ due to scattering processes. $I\left\{n_{p}\right\}$ will be discussed in detail in the next section, and we mention here only that it conserves momentum and energy, namely

$$
\sum_{p} E_{p} I\left\{n_{p}\right\}=0=\sum_{p} \mathbf{p} I\left\{n_{p}\right\}
$$

Note that particle conservation is not a consequence of the equations, but is an independent equation of the theory, reflecting the fact that we had to choose a certain gauge to be able to derive the transport equation (only in the gauge in which the order parameter is real is a gradient expansion possible). Thus we have to add the continuity equation:

$$
\dot{\rho}+\nabla \cdot \mathbf{j}=0
$$

We study now in more detail the energy as given in Eqs. (14) and (19), and consider it as a function of $\phi, \mathbf{v}_{s}, \sigma_{11}, \sigma_{12},\left\{n_{p}\right\}$, and $n_{0}$. If we minimize $e$ with respect to $\sigma_{11}$ and $\sigma_{12}$, we obtain just the correct mean field self-energy equations; $\rho=-\partial e / \partial \phi$ leads to the correct expression for the total density, as given in (22):

$$
\sigma_{11}=2 v \rho ; \quad \sigma_{12} / v=n_{0}-\sum_{p} u_{p} v_{p}\left(2 n_{p}+1\right)
$$


As an important point, we emphasize that the number of condensed particles $n_{0}$ is still a free variable. From the condition that $e$ should be minimal with respect to $n_{0}$, we obtain the last equation:

$$
\sigma_{11}+\sigma_{12}=\phi+2 n_{0} v
$$

Of course, the above considerations remind one of typical equilibrium arguments, where in addition $n_{p}$ should equal the Bose function (which follows from the requirement that $e-T s$ must be minimal with respect to $n_{p}$ ). However, we may turn the argument around: Consider any nonequilibrium situation (within the limits of the theory, of course), and start from the Boltzmann equation, Eq. (24), the continuity equation, and the expression for the energy. From the requirement that the conservation laws are a consequence, we find that the self-energy equations are of the correct form, and, in addition, that (10) and especially (28) must hold. Consequently, Eqs. (10), (22)-(24), and (26)-(28) form the basis of the kinetic theory.

Finally, the momentum and energy conservation laws are obtained in the following form:

$$
\begin{aligned}
\partial_{t} j_{i}+\nabla_{j} \pi_{i j} & =\rho\left(F_{x}\right)_{i} / m \\
\partial_{t}\left(e+\rho \phi_{0}\right)+\nabla \cdot\left(\phi_{0} \mathbf{j}+\mathbf{j}_{e}\right) & =\mathbf{j} \cdot \mathbf{F}_{x}
\end{aligned}
$$

with $\phi_{0}=\phi+\frac{1}{2} m v_{s}^{2}$, and

$$
\begin{aligned}
& \pi_{i j}= \rho\left(v_{s}\right)_{i}\left(v_{s}\right)_{j}+\left(v_{s}\right)_{i}\left(\dot{j}_{1}\right)_{j}+\left(v_{s}\right)_{j}\left(j_{1}\right)_{i} \\
&+\sum_{p} \frac{\partial E_{p}}{\partial p_{j}} \frac{p_{i}}{m} n_{p}+\frac{1}{m} \delta_{i j}\left(\sum_{p} \check{E}_{p} n_{p}-e\right) \\
& \mathbf{j}_{e}=\sum_{p} \check{E}_{p} \frac{\partial \check{E}_{p}}{\partial \mathbf{p}} n_{p}
\end{aligned}
$$

The kinetic equations can be generalized to include, as a formal device, an external field $\eta_{x}$ which couples directly to the order parameter. In Eq. (6), this will introduce an additional term of the form $\eta_{x} \bar{\psi}^{+}+\eta_{x}^{*} \bar{\psi}$; proceeding in the same way as above, we then find an additional term in the energy density, Eq. (14), given by $2 n_{0}^{1 / 2} \eta_{x}^{\mathrm{L}}$, where

$$
\eta_{x}^{\mathrm{L}}=\operatorname{Re} e^{-i x} \eta_{x} ; \quad \eta_{x}^{\mathrm{T}}=\operatorname{Im} e^{-i x} \eta_{x}
$$

$\eta_{x}^{\mathrm{L}(\mathrm{T})}$ may be called the longitudinal (transverse) part of $\eta_{x}$ with respect to the direction of the order parameter considered as a vector in the complex plane. Consequently, the conservation laws appear in slightly modified form, since $e$ and thus $\pi_{i j}$ via Eq. (31) are modified. In Eqs. (29) and (30) we find additional terms on the right-hand side, given by $-2 n_{0}^{1 / 2} \nabla \eta_{x}^{\mathrm{L}} / m$ and 
$2 n_{0}^{1 / 2} \partial_{t} \eta_{x}^{\mathrm{L}}$, respectively. Minimizing $e$ with respect to $n_{0}$, furthermore, leads to the condition [instead of (28)]

$$
\sigma_{11}+\sigma_{12}+\eta_{x}^{\mathrm{L}} / n_{0}^{1 / 2}=\phi+2 n_{0} v
$$

The transverse part of the field, however, appears only in the continuity equation, which now has the following form

$$
\dot{\rho}+\nabla \cdot \mathbf{j}=2 n_{0}^{1 / 2} \eta_{x}^{\mathrm{T}}
$$

\section{COLLISION OPERATORS AND SCATTERING RATES}

The collision operator in the transport equation, Eq. (24), can be derived using standard golden rule arguments. As a consequence of the special treatment of the zero-momentum states, Eq. (13), we have to consider two contributions in the interaction, which we denote by $h_{3}$ and $h_{4}$, namely

$$
h_{3}=v n_{0}^{1 / 2} \hat{\varphi}^{+} \hat{\varphi} \hat{\varphi}+\text { H.c. }
$$

and

$$
h_{4}=\frac{1}{2} v \hat{\varphi}^{+} \hat{\varphi}^{+} \hat{\varphi} \hat{\varphi}
$$

$h_{3}$ describes processes in which the excitations interact with the particles of the condensate, while $h_{4}$ is just the scattering of excitations. Accordingly, we find

$$
I\left\{n_{p}\right\}=I_{3}\left\{n_{p}\right\}+I_{4}\left\{n_{p}\right\}
$$

and we are able to define two scattering rates $\tau_{3}^{-1}(p)$ and $\tau_{4}^{-1}(p)$ from the linearized form of $I_{3}$ and $I_{4}$.

\subsection{Three-Excitation Processes}

We find $I_{3}$ to be given by*

$$
\begin{aligned}
I_{3}\left\{n_{p_{1}}\right\}= & -4 \pi n_{0} v^{2} \sum_{p_{2}, p_{3}}\left\{|A(1 ; 2,3)|^{2}\right. \\
& \times\left[n_{1}\left(1+n_{2}\right)\left(1+n_{3}\right)-\left(1+n_{1}\right) n_{2} n_{3}\right] \delta(1-2-3) \\
& +2|A(3 ; 1,2)|^{2} \\
& \left.\times\left[n_{1} n_{2}\left(1+n_{3}\right)-\left(1+n_{1}\right)\left(1+n_{2}\right) n_{3}\right] \delta(1+2-3)\right\}
\end{aligned}
$$

where $n_{1}=n\left(\mathbf{p}_{1}\right)$, etc., and

$$
\delta(1-2-3)=(2 \pi)^{3} \delta\left(\mathbf{p}_{1}-\mathbf{p}_{2}-\mathbf{p}_{3}\right) \delta\left(E_{1}-E_{2}-E_{3}\right)
$$

*We use units such that $\hbar=k_{\mathrm{B}}=1$ in the following. 
The scattering amplitude $A$ is obtained in terms of the coherence factors $u$ and $v$, with the result

$$
A(1 ; 2,3)=u_{1} u_{2} u_{3}-u_{1} u_{2} v_{3}-u_{1} v_{2} u_{3}+v_{1} v_{2} u_{3}+v_{1} u_{2} v_{3}-v_{1} v_{2} v_{3}
$$

As an illustration, consider the Bogoliubov approximation, and define $p_{0}^{2}=2 m n_{0} v$, i.e., $p_{0}$ is the characteristic momentum for the crossover between the linear and the quadratic part of the spectrum. Then it is clear that $A \rightarrow 1$ if all momenta are $\gg p_{0}$, in which case $u \rightarrow 1, v \rightarrow 0$ (normal state limit). On the other hand, if all three momenta are small, $\ll p_{0}$, we find

$$
A(1 ; 2,3)=A(3 ; 1,2) \approx \frac{3}{2^{7 / 4}}\left(\frac{p_{1} p_{2} p_{3}}{p_{0}^{3}}\right)^{1 / 2}
$$

provided the energies of the excitation are related by the appropriate conservation law, $E_{1}=E_{2}+E_{2}$ and $E_{1}+E_{2}=E_{3}$, respectively. In addition, we also have to consider the scattering of low-momentum excitations ("phonons") with high-momentum excitations ("particles"). The corresponding amplitude is given by

$$
A(1 ; 2,3) \approx 2^{3 / 4}\left(p_{3} / p_{0}\right)^{1 / 2}
$$

for $p_{1}, p_{2} \gg p_{0} ; p_{3} \ll p_{0}$.

In the limit of low temperatures, $T \ll n_{0} v$, and in the Bogoliubov approximation, the scattering rate can be evaluated. We find

$$
\begin{aligned}
& \frac{1}{\tau_{3}}(p)= \frac{3}{320 \pi m n_{0}} p^{5}+\frac{3 \pi m^{3} T^{4}}{320 n_{0}^{3} a^{2}} p \\
&+ \frac{9 m^{4} T^{5}}{256 \pi^{7 / 2} a^{5 / 2} n_{0}^{7 / 2}}\left[z^{2} D_{2}(z)-2 z D_{3}(z)+D_{4}(z)\right] \\
& E_{p}, T \ll n_{0} v
\end{aligned}
$$

with $z=E_{p} / T$, and

$$
D_{n}(z)=\int_{0}^{z} d x \frac{x^{n}}{e^{x}-1}
$$

We easily identify the first two terms, which dominate for $E_{p} \gg T$ and $E_{p} \ll T$, respectively, as the terms calculated already by Belyaev ${ }^{7}$ and Mohling and Morita. ${ }^{22}$ For $E_{p} \leqslant T$, we find that the Mohling-Morita term dominates (numerically), and thus obtain the rather simple approximation

$$
\frac{1}{\tau_{3}}(p) \approx\left(\frac{l}{a}\right)^{2}\left(\frac{T}{T_{c}}\right)^{4}(p l) T_{c}
$$

provided $E_{p} \leqslant T$ and $T \ll n_{0} v$. Note that the latter condition corresponds 
to $T / T_{c} \ll a / l$, i.e., to very low temperatures; also, $E_{p} \approx c p$, where $c^{2}=$ $n_{0} v / m$ is the phonon velocity in the Bogoliubov approximation; and we have used $\rho=l^{-3}=n_{0}$ since we can neglect the small ground-state depletion.

We emphasize that in arriving at the above result, we have to evaluate very carefully the energy $\delta$-functions, and to take the deviation from the linear spectrum into account. Consequently, the change in the momentum of the colliding phonons turns out to be very small, of order $T / n_{0} v$ only. We thus have to interpret the above result as the energy relaxation rate, and the momentum relaxation rate will be even smaller.*

In the high-temperature limit, $T \gg n_{0} v$, on the other hand, the scattering rate cannot be determined exactly. In evaluating certain integrals, we use the following approximate procedure: We split the range of integration into "high" and "low" momenta, i.e., $p \geqslant p_{0}$ and $p \leqslant p_{0}$, and use the expressions for the scattering amplitude and the spectrum as obtained in the limits $p \gg p_{0}$ and $p \ll p_{0}$, respectively (in the Bogoliubov approximation). It turns out that, apart from numerical factors, the integrals over high and low momenta give the same answer, giving us some confidence in the following results. Also, as far as the momentum dependence is concerned, we find that different regimes join smoothly. We obtain

$$
\begin{aligned}
& T \gg n_{0} v: \\
& \frac{1}{\tau_{3}}(p) \approx \begin{cases}\frac{8 T m v}{\pi} p \sim \frac{a}{l} T(p l), & p \ll p_{0} \\
\frac{T m^{2} n_{0} \vartheta^{2}}{p} A \sim \frac{n_{0}}{\rho}\left(\frac{a}{l}\right)^{2} \frac{T}{p l}, & p_{0} \ll p \ll p_{T} \\
\frac{m n_{0} v^{2}}{2 \pi} p \sim \frac{n_{0}}{\rho}\left(\frac{a}{l}\right)^{2}(p l) T_{c}, & p_{T} \ll p\end{cases}
\end{aligned}
$$

We define here the thermal momentum $p_{T}$ by $E\left(p_{T}\right)=T$, and the quantity $A$ is given by

$$
A \approx \frac{2}{\pi}+\frac{7}{2 \pi} \log \frac{2 E_{p}}{n_{0} v^{2}}
$$

Although for detailed calculations the momentum dependence of the scattering rate has to be taken into account very carefully, it is often convenient to discuss relaxation processes in terms of the scattering time, evaluated for certain characteristic $p$. Clearly, the important momenta at

*Compare with the results for the scattering of electrons by phonons in metals at low temperatures. In that case, the momentum relaxation rate is smaller than the energy relaxation rate by a factor $\left(T / \theta_{\mathrm{D}}\right)^{2}$, where $\theta_{\mathrm{D}}$ is the Debye temperature. 
low temperatures are given by $p_{T}=T / c$, and Eq. (46) leads to

$$
\frac{1}{\tau_{3}}\left(p_{T}\right) \approx\left(\frac{l}{a}\right)^{5 / 2}\left(\frac{T}{T_{c}}\right)^{5} T_{c}, \quad T \ll \frac{a}{l} T_{c}
$$

which leads to the numerical value $\tau_{3}\left(p_{T}\right) \sim 10^{-5} \mathrm{sec}$ for $a / l \approx 10^{-2}, T \approx$ $10^{-2} T_{c}, T_{c} \approx 70 \mathrm{mK}$. For high temperatures, we find two characteristic times, $\tau_{3}\left(p_{0}\right)$ and $\tau_{3}\left(p_{T}\right)$, the former determining the time for relaxation processes in the phonon part of the spectrum, and the latter giving the time for thermalizing the quasiparticles. We obtain from (47)

$$
\begin{gathered}
\frac{1}{\tau_{3}}\left(p_{0}\right) \sim 10^{2}\left(\frac{n_{0}}{\rho}\right)^{1 / 2}\left(\frac{a}{l}\right)^{3 / 2} \frac{T}{T_{c}} T_{c} \\
\frac{1}{\tau_{3}}\left(p_{T}\right) \sim 10^{2} \frac{n_{0}}{\rho}\left(\frac{a}{l}\right)^{2}\left(\frac{T}{T_{c}}\right)^{1 / 2} T_{c} \\
\frac{a}{l} T_{c} \ll T
\end{gathered}
$$

and realize that

$$
\tau_{3}\left(p_{T}\right) \sim \tau_{3}\left(p_{0}\right)\left(\frac{\rho}{n_{0}} \frac{l}{a} \frac{T}{T_{c}}\right)^{1 / 2}
$$

i.e., $\tau_{3}\left(p_{T}\right) \gg \tau_{3}\left(p_{0}\right)$. For $T \approx T_{c} \approx 70 \mathrm{mK}$ and $a / l \approx 10^{-2}$, we find $\tau_{3}\left(p_{T}\right) \approx$ $10^{-8}\left(\rho / n_{0}\right)$ sec. Note that Eqs. (49) and (50) agree for $T / T_{c} \approx 3 a / l$.

\subsection{Four-Excitation Processes}

As an illustration, we consider first the collision operator in the normal state. The result is well known:

$$
\begin{aligned}
I_{4}^{\mathrm{N}}\left\{n_{p_{1}}\right\}= & -4 \pi v^{2} \sum_{p_{2}, p_{3}, p_{4}} \delta(1+2-3-3)|A(1,2 ; 3,4)|^{2} \\
& \times\left[n_{1} n_{2}\left(1+n_{3}\right)\left(1+n_{4}\right)-\left(1+n_{1}\right)\left(1+n_{2}\right) n_{3} n_{4}\right]
\end{aligned}
$$

and we use an obvious generalization of our previous notation. $I_{4}^{\mathrm{N}}$ describes scattering processes of the form $1+2 \rightarrow 3+4$, and $|A|^{2}=1$ in the normal state. Below the transition temperature, we obtain, in addition to a contribution of the same structure as (53), also terms describing processes like $1 \rightarrow 2+3+4$ and $1+2+3 \rightarrow 4$. We again use the approximate procedure described above to calculate the corresponding scattering rates. The transition probabilities in the limit where all momenta are $\gg p_{0}$ are easily calculated, and we find $|A(1,2 ; 3,4)|^{2} \approx 1$ (as expected) and $|A(1,2,3 ; 4)|^{2} \approx v_{2}^{2}$, which is of order $\left(n_{0} v / T\right)^{2}$ for thermal energies. We thus conclude that the 
contributions to $I_{4}$ besides $I_{4}^{\mathrm{N}}$ are small and can be neglected compared to $I_{3}$. (In the low-temperature regime, it has been assumed by many authors that four-phonon processes can be neglected compared to three-phonon processes.) We point out, however, a difficulty that arises in the phonon regime. If all momenta are much less than $p_{0}$, we find the unphysical result $|A|^{2} \approx\left(p_{1} p_{2} p_{3} p_{4}\right)^{-1}$, while phenomenological approaches ${ }^{14}$ predict, as in (42) and (43), the behavior $p^{+1}$ for every phonon. This behavior may be connected with the divergences discussed in Refs. 11 and 12, and the correct result for three-phonon processes seems surprising at this point. An investigation of this question is not intended here, and we use in the following $I_{4}^{\mathrm{N}}$ with $|A|^{2}=1$ : namely, it turns out that we need to consider $I_{4}$ only in the high-temperature region where $n_{0} \ll \rho$, and in addition only for thermal momenta, $p \approx p_{T}$.

The scattering rate, as calculated from $I_{4}^{\mathrm{N}}$ with $|A|^{2}=1$, is obtained as described in the limit $T \gg n_{0} v$. We find it to be roughly independent of momentum in the range $p_{0} \ll p \leqslant p_{T}$, and to increase linearly in $p$ for $p \geqslant p_{T}$. The result is

$$
\frac{1}{\tau_{4}}(p) \sim 20\left(\frac{a}{l}\right)^{2}\left(\frac{T}{T_{c}}\right)^{2}\left(1+\frac{p}{p_{T}}\right) T_{c}
$$

provided $p \gg p_{0}$. For $T \approx T_{c}$ and the considered parameter values, we find as the characteristic time $\tau_{4}\left(p_{T_{c}}\right) \approx 10^{-7} \mathrm{sec}$. We also conclude from (51) and (54) that $\tau_{4}\left(p_{T}\right) \geqslant \tau_{3}\left(p_{T}\right)$ provided $2 n_{0} / \rho \geqslant\left(T / T_{c}\right)^{3 / 2}$. This result has the simple interpretation that $I_{3}$ dominates over $I_{4}$ (for thermal momenta) when the number of the condensed atoms is larger than the number in the excited states. Using the ideal gas result for $n_{0}(T)$, we estimate that the three-excitation processes $\left(I_{3}\right)$ dominate for $T \leqslant 0.7 T_{c}$.

\section{RELAXATION OF NONEQUILIBRIUM STATES}

An important feature of the conserving approximation is the appearance of a gap in the excitation spectrum, Eq. (21). As an illustration, consider the equations in equilibrium. With the help of (28) and $\sigma_{11}=2 v \rho$ we find

$$
\Delta^{2}=4 n_{0} \vartheta\left(n_{0} \vartheta-\sigma_{12}\right)
$$

i.e., the gap is related to the difference between $\sigma_{12}$ and $n_{0} v$. Inserting the equilibrium distribution function into (27), we obtain

$$
n_{0} v-\sigma_{12}=v \sigma_{12} \sum_{p}\left(\frac{1}{2 E_{p}} \operatorname{cth} \frac{E_{p}}{2 T}-\frac{m}{p^{2}}\right)
$$


where we subtracted $m / p^{2}$ to regularize the divergence for large-momenta [which can be interpreted in the sense of Eq. (1)]. Since $n_{0} v-\sigma_{12}$ is expected to be small, we put $\Delta=0$ on the right-hand side of Eq. (56), and obtain

$$
\left(\frac{\Delta}{n_{\mathrm{c}} v}\right)^{2} \sim\left\{\begin{array}{cl}
\left(\frac{a}{l}\right)^{1 / 2} \frac{T}{T_{c}}\left(\frac{\rho}{n_{0}}\right)^{1 / 2}, & n_{0} v \ll T \\
\left(\frac{a}{l}\right)^{3 / 2}, & T \ll n_{0} \nu
\end{array}\right.
$$

So we certainly have $\Delta \ll n_{0} v$ provided $n_{0} / \rho \geqslant a / l$, i.e., except in the critical region close to $T_{c}$. We also find that $\Delta \ll T$ as long as the temperature is not extremely small, $T / T_{c} \geqslant(a / l)^{7 / 4}$. Consequently, we will exclude very low temperatures as well. For later use, we define $p_{\Delta}=\Delta / c$, i.e., $p_{\Delta} / p_{0} \approx$ $\Delta / n_{0} v$. Note that, for $\Delta \rightarrow 0$, the spectrum is phononlike with velocity $c^{2} \approx n_{0} v / m$, the Bogoliubov result.

We want to determine now the relaxation rate of a nonequilibrium distribution and imagine a time-dependent process in which initially the equilibrium between condensed atoms and excitations is slightly disturbed. We consider a spatially homogeneous situation, without any currents, and assume the deviations from equilibrium to be small. We then linearize the equations according to $n_{p}=b\left(E_{p}\right)+\delta n_{p}$, where $b\left(E_{p}\right)$ is the Bose function at the unperturbed energy, $\phi \rightarrow \phi+\delta \phi, n_{0} \rightarrow n_{0}+\delta n_{0}, \rho \rightarrow \rho+\delta \rho$; and we will use in a moment that the total density is constant in the present situation: $\delta \rho=0$. From (24), (22), and (27) we find, after eliminating $\delta \sigma_{12}$ by (28), the following equations $(\partial / \partial t \rightarrow-i \omega)$ :

$$
\begin{gathered}
-i \omega \delta n_{p}=I\left\{b+\delta n_{p}\right\} \\
v\left(\delta n_{0}-\delta \rho\right)+K_{1}=-\vartheta \sum_{p} \frac{\varepsilon_{p}}{E_{p}} \delta n_{p} \\
v \delta n_{0}+\delta \phi-2 \vartheta \delta \rho+K_{2}=-v \sum_{p} \frac{\sigma_{12}}{E_{p}} \delta n_{p}
\end{gathered}
$$

with

$$
\begin{aligned}
& K_{1}=v \sum_{p} \delta\left(u_{p}^{2}\right) \operatorname{cth}\left(E_{p} / 2 T\right) \\
& K_{2}=v \sum_{p} \delta\left(u_{p} v_{p}\right) \operatorname{cth}\left(E_{p} / 2 T\right)
\end{aligned}
$$

$\delta\left(u_{p}^{2}\right)$ and $\delta\left(u_{p} v_{p}\right)$ can be calculated easily from the definition of the coherence factors, Eq. (18). Equations (58)-(60) form a closed set of equations: It must be emphasized that $I$ has the property that it relaxes 
$\delta n_{p}$ to a local equilibrium function, i.e.,

$$
\delta E_{p}=\left(\partial E_{p} / \partial n_{0}\right) \delta n_{0}+\left(\partial E_{p} / \partial \phi\right) \delta \phi+\left(\partial E_{p} / \partial \rho\right) \delta \rho
$$

appears as a "drive term" in the Boltzmann equation. This becomes more evident if we linearize in a different way, namely according to $n_{p}=$ $b\left(E_{p}+\delta E_{p}\right)+\delta \bar{n}_{p}\left(\delta \bar{n}_{p}\right.$ describes the deviation from local equilibrium). In principle, we can determine now $\delta n_{p}$ in terms of $\delta n_{0}, \delta \phi$, and $\delta \rho$ from (58), and then solve (59) and (60) to find the relaxation rate $i \omega \equiv \lambda$.

However, things can be simplified a bit more. First, we consider $K_{1}$ and $K_{2}$; we notice that the integrals are infinite due to the long-wavelength behavior if we use the Bogoliubov approximation (the divergence is of the form $\sum_{p} p^{-4}$ ). In the approximation with a gap, on the other hand, we find that $K_{1}$ and $K_{2}$, although proportional to $p_{\Delta}^{-1}$, are negligible in (59) and (60) except close to $T_{c}$. Second, we find it convenient to use the gap and the phonon velocity as variables [see Eq. (21)]:

$$
\begin{aligned}
m \delta c^{2} & =2 \nu \delta \rho-\delta \phi \\
-\frac{1}{2} \Delta \delta \Delta & =\sigma_{12} v \delta n_{0}+n_{0} v(\delta \phi-2 v \delta \rho)
\end{aligned}
$$

Note that

$$
\delta E_{p}=\frac{\Delta \delta \Delta}{E_{p}}+\frac{\xi_{p}}{E_{p}} m \delta c^{2}
$$

Neglecting $K_{1}, K_{2}$, and small corrections $\sim \Delta^{2}$, we can rewrite (59) and $(60)$ in the following form:

$$
\begin{aligned}
m \delta c^{2}-\vartheta \delta \rho & \approx-v \sum_{p} \frac{\xi_{p}}{E_{p}} \delta n_{p} \approx\left(2 n_{0}\right)^{-1} \sum_{p} \frac{\xi_{p}^{2}}{E_{p}} \delta n_{p} \\
\frac{\Delta \delta \Delta}{2 n_{0} v} & \approx v \sum_{p} \frac{n_{0} v}{E_{p}} \delta n_{p}
\end{aligned}
$$

We used the fact that energy is conserved in collisions, i.e., $\sum_{p} E_{p} \delta n_{p}=0$, to establish the second equality in (63). The last expression in (63) is convenient at low temperatures, and the other one at high temperatures: Namely, it is straightforward to establish the following inequalities $(\delta \rho=0)$ :

$$
\begin{array}{ll}
m \delta c^{2} \ll \frac{\Delta \delta \Delta}{n_{0} v} & \text { for } \quad T \ll \frac{a}{l} T_{c} \\
m \delta c^{2} \gg \frac{\Delta \delta \Delta}{n_{0} \vartheta} & \text { for } \quad T \gg \frac{a}{l} T_{c}
\end{array}
$$

since the relevant energies turn out to be of order $T$ in the integrals. In 
addition, we then conclude that $\Delta \delta \Delta / 2 n_{0} \approx \approx-v \delta n_{0}$ and $m \delta c^{2} \approx v \delta n_{0}$ for low and high temperatures, respectively. Consequently, we arrive at two equations, which can be combined into a single one, namely

$$
\delta n_{0} \approx-\sum_{p} \frac{\xi_{p}+n_{0} v}{E_{p}} \delta n_{p}
$$

since $\xi_{p} \ll n_{0} v\left(\xi_{p} \gg n_{0} v\right)$ for low (high) $T$. Equation (67) agrees with the naive use of the Bogoliubov approximation, i.e., Eq. (2) together with Eq. (22), in which case it is obvious that $m \delta c^{2}=v \delta n_{0}$. Note also that, in a situation where $\delta n_{p}$ describes a shift in temperature, i.e.,

$$
\delta n_{p}=\left(-b^{\prime}\right) \frac{E_{p}}{T} \delta T
$$

Eq. (67) reduces to the equilibrium relation between the number of condensed particles and the temperature, at constant total density. Clearly, in the Bogoliubov approximation, we find $\partial n_{0} / \partial T \sim-T$ at low temperatures, and $\partial n_{0} / \partial T \sim-T^{1 / 2}$ at high temperatures, which is just the ideal gas result.

\subsection{Relaxation at Low and Intermediate Temperatures: $T \leq 0.7 T_{c}$}

To determine the relaxation rate, we finally have to solve the Boltzmann equation. We introduce an approximation for the collision operator $I$ that takes into account that (a) $I$ relaxes toward local equilibrium while (b) conserving energy. A relaxation time approximation taking care of these properties is usually a good approximation for low frequencies (and long wavelengths), i.e., in the hydrodynamic regime, although we expect reasonable results for $\omega$ of the order of the scattering rate. However, the situation seems to be more complicated for $T \rightarrow T_{c}$, where $I_{3}$ and $I_{4}$ have to be taken into account (see the following section).

In this approximation, the Boltzmann equation reduces to

$$
-i \omega \delta n_{p}=-\frac{1}{\tau_{p}}\left\{\delta n_{p}-\left(-b^{\prime}\right)\left[\frac{E_{p}}{T} \delta T-\delta E_{p}\right]\right\}
$$

where $b^{\prime}=\partial b / \partial E_{p}$. The change in temperature $\delta T$ is determined from energy conservation. Evidently, we can solve (69) easily for $\delta n_{p}$ : As a formal device, we introduce $\alpha[\cdot]$ by $(\lambda=i \omega)$

$$
\alpha[\cdot]=v \sum_{p}\left(1-\lambda \tau_{p}\right)^{-1}\left(-b^{\prime}\right)[\cdot]
$$

and obtain from (67) and (69)

$$
v \delta n_{0}=-\alpha\left[\varepsilon_{p}\right] \frac{\delta T}{T}+\alpha\left[\frac{\varepsilon_{p}}{E_{p}} \delta E_{p}\right]
$$




$$
\alpha\left[E_{p}^{2}\right] \frac{\delta T}{T}=\alpha\left[E_{p} \cdot \delta E_{p}\right]
$$

Being a bit more careful, we have to replace $\varepsilon_{p}$ by $n_{0} v\left(\xi_{p}\right)$ for low (high) temperatures in Eq. (71). In addition, we emphasize that $\delta E_{p} \approx$ $-\left(2 n_{0} v / E_{p}\right) v \delta n_{0}$ and $\delta E_{p} \approx\left(\xi_{p} / E_{p}\right) v \delta n_{0}$ in the respective regimes. We realize now that we may put $\Delta=0$ except in $\alpha\left[E_{p}^{-2}\right]$, where we obtain a logarithmic dependence on $p_{\Delta}$. Due to the smallness of $\alpha[\cdot]$, e.g., for $\lambda=0$ :

$$
\alpha[1] \sim \begin{cases}v \frac{p_{T}^{3}}{T} \sim\left(\frac{a}{l}\right)^{3 / 2}\left(\frac{T}{n_{0} v}\right)^{2}, & T \ll n_{0} v \\ v p_{0}^{3} \frac{T}{\left(n_{0} v\right)^{2}} \sim\left(\frac{a}{l}\right)^{3 / 2} \frac{T}{n_{0} v}, & T \gg n_{0} v\end{cases}
$$

we conclude that the solution of (71) and (72) is characterized by $\delta T \gg v \delta n_{0}$, i.e., the eigenfunction is dominated by a shift in temperature. The eigenvalue $\lambda$ is thus determined by $\alpha\left[E_{p}^{2}\right] \approx 0$, and will be of the order of the quasiparticle relaxation rate evaluated for thermal momenta. Using the expressions for $\tau_{p}$ we obtained earlier, i.e., Eq. (46) and the intermediate range result of Eq. (47), $\tau_{p} \sim p$, respectively, we find (numerically) the result

$$
\lambda \approx \begin{cases}5 / \tau_{3}\left(p_{T}\right), & T \leqslant(a / l) T_{c} \\ 0.6 / \tau_{3}\left(p_{T}\right), & (a / l) T_{c} \leqslant T \leqslant 0.7 T_{c}\end{cases}
$$

$p_{T}$ is defined by $E\left(p_{T}\right)=T$; note that $\tau_{3}\left(p_{T}\right)$ is different in the two regimes. Physically, this result expresses the fact that the long-time behavior is dominated by the time characteristic for the thermalization of the quasiparticles, which thus sets the scale for the time in which the number of condensed atoms $n_{0}$ approaches its equilibrium value. However, we hesitate to call this time the "order parameter relaxation time" since no characteristics of the order parameter are involved. To substantiate this conclusion, we consider the linear response to a field that couples directly to the order parameter, $\eta_{x}$, as introduced in Section 2. For frequencies not too close to the one given in Eq. (74), we find that the coupling to the quasiparticles is negligible $(\alpha[\cdot] \ll 1)$, and obtain

$$
\begin{aligned}
m \delta c^{2} & \approx-\eta_{x}^{\mathrm{L}} / n_{0}^{1 / 2}+2 v n_{0}^{1 / 2} \eta_{x}^{\mathrm{T}} /(-i \omega) \\
\Delta \delta \Delta / 2 n_{0} \vartheta & \approx-\delta \eta_{x}^{\mathrm{L}} / 2 n_{0}^{1 / 2}
\end{aligned}
$$

in agreement with the results of Appendix B, since (75) and (76) can also be written as

$$
\delta \phi \approx \eta_{x}^{\mathrm{L}} / n_{0}^{1 / 2}+\vartheta \delta \rho, \quad \delta n_{0} \approx \delta \rho
$$


with $\delta \rho$ related to the transverse part of the external field, $\delta \rho=$ $2 n_{0}^{1 / 2} \eta_{x}^{\mathrm{T}} /(-i \omega)$. Of course, the relaxation rate calculated above appears as a pole in the order parameter susceptibilities; it turns out, however, that the residuum of the pole is of the order $(a / l)^{3 / 2}$ and $(a / l)\left(T / T_{c}\right)^{1 / 2}$, respectively, at low and high temperatures.

We have not investigated the equations for higher frequencies, say $\omega \sim 1 / \tau\left(p_{0}\right)$, in great detail since (a) the momentum dependence of $\tau_{p}$ is not known accurately enough, and (b) the simple approximation for the collision operator may be questionable. However, we expect, at least for $T \gg n_{0} v$, that a characteristic relaxation occurs on the scale $\tau\left(p_{0}\right)$, i.e., fast compared to the thermalization time of the quasiparticles, and thus dominated by strong nonequilibrium states. Thus the relaxation appears as a two-stage process. Starting from an arbitrary initial distribution, we have at first a fast process, with time scale $\tau\left(p_{0}\right)$, characterized by rearrangements of the excitations at small energies, $\leqslant n_{0} v \ll T$, with changes of $\Delta^{2}$ and $c^{2}$ being of equal importance. In the next step, the excitations have to be thermalized, which takes a rather long time, $\tau\left(p_{T}\right)$. From this discussion, it is tempting to identify $\tau\left(p_{0}\right)$ as the order parameter relaxation time (and we have done $\mathrm{so}^{21}$ ); however, it appears difficult to support this conjecture within the simple approximations we are studying here. The comparison with analogous situations in superconductors is instructive in this context (see Section 5).

\subsection{Relaxation close to $T_{c}$}

As discussed in detail in Section 3, we have to include four-excitation processes in the high-temperature region: $I=I_{3}+I_{4}^{\mathrm{N}}$, where $I_{4}^{\mathrm{N}}$ is the part of $I_{4}$ that has the same structure as the normal state collision operator (it describes processes of the form $1+2 \rightarrow 3+4$ ). While, of course, $I_{3}$ and $I_{4}^{\mathrm{N}}$ conserve energy individually, there is an important difference: $I_{4}^{\mathrm{N}}$ also conserves the number of excitations! This follows directly from (53), and can be expressed as

$$
\sum_{p} I_{4}^{\mathrm{N}}\left\{n_{p}\right\}=0
$$

Also, for $T \rightarrow T_{c}$, the number of excitations equals the number of atoms in the excited states: we have $u_{p}^{2} \rightarrow 1$ and $v_{p}^{2} \rightarrow 0$ in this limit. Thus we conclude that $I_{4}^{N}$ cannot contribute to establishing the equilibrium with respect to the particle number between condensed and excitated atoms; it is only effective in thermalizing the excitations. Consequently, we have three time scales: $\tau_{3}\left(p_{0}\right) \ll \tau_{4}\left(p_{T}\right) \ll \tau_{3}\left(p_{T}\right)$, and interpret the relaxation process in the following way. As discussed in the previous section, we have a fast process characterized by changes for low momenta, $\sim \tau_{3}\left(p_{0}\right)$. Then it takes a time 
of order $\tau_{4}\left(p_{T}\right)$ to thermalize the excitations, and, finally, the equilibrium between condensed and excited atoms is established within a time of the order of $\tau_{3}\left(p_{T}\right)$. We conclude again that $\tau_{3}\left(p_{T}\right)$ determined the long-time behavior of the relaxation, and thus we find for all temperatures $\lambda \sim \tau_{3}^{-1}\left(p_{T}\right)$. From Eq. (51), and using the ideal gas result for $n_{0}(T)$, we find close to $T_{c}$

$$
\lambda \approx 10^{2}\left(\frac{a}{l}\right)^{2} \varepsilon T_{c}, \quad \varepsilon=\frac{T_{c}-T}{T_{c}} \ll 1
$$

and we can also express this in the form $\lambda \sim \varepsilon / \tau_{N}$, where $\tau_{N}=\tau_{4}\left(p_{T_{c}}\right)$ is the characteristic relaxation time in the normal state at the critical temperature. Note, however, that the critical region was excluded in our considerations, i.e., $\varepsilon \geqslant a / l$.

\section{SUMMARY AND DISCUSSION}

In conclusion, we have studied the kinetic equations for a dilute Bose gas. The kinetic theory consists of the self-consistent mean field equations plus a Boltzmann equation from which the distribution function of excitations has to be calculated. It applies to long-wavelength, low-frequency situations and assumes the quasiparticles to be well defined: the latter condition leads to the conclusion that the critical region near $T_{c}, \varepsilon \leqslant a / l$, has to be excluded. Gould and Wong ${ }^{17}$ have argued that, in a second-order approximation, one is able to handle temperatures such that $(a / l)^{2} \leqslant$ $\varepsilon \leqslant a / l$.

In particular, we considered the relaxation of a nonequilibrium distribution of the quasiparticles. We found that the relaxation time is determined by $\tau_{3}\left(p_{T}\right)$, i.e., the relaxation time for three-excitation processes evaluated for thermal energies. For not too low temperatures $\left(T / T_{c} \geqslant a / l \approx 10^{-2}\right)$, we obtained the following result:

$$
\lambda \approx \frac{1}{\tau_{3}\left(p_{T}\right)} \approx \frac{n_{0}}{\rho}\left(\frac{T}{T_{c}}\right)^{1 / 2} \times 10^{8} \sec ^{-1}
$$

where $T_{c} \approx 70 \mathrm{mK}$ was inserted. Note that $n_{0} / \rho \approx \varepsilon=\left(T_{c}-T\right) / T_{c}$. This result has a very simple interpretation at intermediate temperatures, $T \leqslant$ $0.7 T_{c}$ : In this regime, $\tau_{3}\left(p_{T}\right)$ is just the thermalization time of the quasiparticles. Imagine a perturbation that initially changes the energy of the excitations: since energy is conserved in the following relaxation, the temperature as well as the number of condensed particles will approach their new equilibrium values on a scale set by $\tau_{3}\left(p_{T}\right)$, of course a plausible result. 
The situation becomes more complicated for high temperatures, $T \geqslant$ $0.7 T_{c}$. To be definite, consider $\varepsilon \ll 1$ (but $\varepsilon \gg 10^{-2}$ ): In this regime, the number of excitations almost equals the number of the particles in the excited states. In addition, the dominant scattering processes will be due to four-excitation processes, which are number conserving for $T \rightarrow T_{c}$; the number-nonconserving contributions to $I_{4}$ are expected to be small (of the order of* $\left.n_{0} v / k_{\mathrm{B}} T_{c}\right)$. Thus the relaxation has two stages: the relatively fast thermalization at constant number of excitations, on a scale $\tau_{4}\left(p_{T}\right)$, followed by the conversion of excitations to condensed particles through threeexcitation processes, i.e., on a scale $\tau_{3}\left(p_{T}\right)$. Since $\tau_{3}\left(p_{T}\right) \approx \tau_{4}\left(p_{T}\right) / \varepsilon, I_{3}$ dominates over the number-nonconserving contributions from $I_{4}$.

In contrast to the Bose gas, relaxation processes and especially the order parameter relaxation in superconductors have been the subject of many investigations, experimental as well as theoretical. $\dagger$ The origin of superconductivity, as well as the (in many metals) dominant scattering mechanism, is due to the electron-phonon interaction. In addition, the phonons can often be considered to be in equilibrium, due to very effective coupling to the surroundings, and act as a heat bath for the electrons. We consider first very low temperatures, and imagine a situation in which initially the electrons are in the (unstable) normal state. As is well known, $;$ "Cooper pairs" will form on a time scale given by $\hbar / k_{\mathrm{B}} T_{c}$, and a gap in the quasiparticle spectrum is established (the gap $\Delta_{s}$ is of the order of $k_{\mathrm{B}} T_{c}$ ). Besides leading to the characteristic equilibrium properties of superconductors, the gap also manifests itself in the high-frequency response: ${ }^{26,27}$ Physically, "pair breaking" is possible when the external frequency exceeds $2 \Delta_{s} / \hbar$. Considering, e.g., the response to a steplike increase of the supercurrent, one finds ${ }^{27}$ a behavior characterized by oscillations with frequency $2 \Delta_{s} / \hbar$ and by algebraic decay in time. For times long compared to the inverse of the gap, electron-phonon scattering becomes important in establishing the final equilibrium state. It is useful to distinguish the scattering time $\tau_{\mathrm{ep}}^{\mathrm{s}}$ and the recombination time $\tau_{\mathrm{ep}}^{\mathrm{r}} \approx \tau_{\mathrm{ep}}^{\mathrm{s}} \exp \left(\Delta_{s} / k_{\mathrm{B}} T\right)$, the latter being related to the recombination of an "electron" and a "hole" under emission of a $2 \Delta_{s}$ phonon (and therefore exponentially long). It turns out that, on a scale set by $\tau_{\mathrm{ep}}^{\mathrm{s}}$, the electrons are thermalized, at a temperature, however, not equal to the bath (phonon) temperature. Thus the long-time behavior is dominated by $\tau_{\mathrm{ep}}^{\mathrm{r}}$, the recombination time, and the gap is time dependent since it adjusts to the actual electron temperature. For this

\footnotetext{
*This small factor is essentially the range in energy over which the coherence factors deviate from their normal state values, divided by the critical temperature. Applying analogous results for superconductors ${ }^{23}$ to the present situation, however, suggests that the factor might be only $\left(n_{0} \nu / k_{\mathrm{B}} T_{c}\right)^{1 / 2}$.

†For general reviews, see Refs. 24 and 25 .

\$See, for example, Chapter 7 of Ref. 16.
} 
reason, $\tau_{\mathrm{ep}}^{\mathrm{r}}$ is sometimes called the order parameter relaxation time. It seems to us, however, that similar reservations as above (see Section 4) should be made.

An important feature of the theory of superconductivity is the smallness of the transition temperature compared to the Fermi temperature, the ratio being of the order of $10^{-4}$. As a consequence, variations of the magnitude and the phase of the order parameter are decoupled (in linear response) in an excellent approximation: Changes of the magnitude couple only to the temperature of the electrons (and the energy), loosely speaking, and changes of the phase to variations of the chemical potential and the density. However, it turns out that, close to the transition temperature, the characteristic time for both modes is of the same order, namely ${ }^{23} \sim \tau_{\mathrm{ep}} / \varepsilon^{1 / 2}$, where $\tau_{\mathrm{ep}}$ is the electron-phonon scattering time in the normal state. Note that the quasiconservation of the number of excitations, which becomes equal to the number of electrons at $T_{c}$, plays an important role in establishing this result for the transverse (phase) mode.

Having some of these results in mind, we have investigated the mean field equations in the collisionless limit. Some details can be found in Appendix B. As already discussed in Section 4, the order parameter response is only weakly modified by the coupling to the quasiparticles. The small corrections, however, turn out to be formally similar to the high-frequency results in superconductors, showing algebraic decay and oscillations with a frequency $2 \Delta / \hbar$. Consequently, these effects depend crucially on the gap in the spectrum, i.e., on the order in perturbation theory in which the calculation is done. We must conclude that the conserving approximation contains effects that must be interpreted with care. However, we emphasize here the smallness of these corrections, which seems related to the fact that Bose condensation is a phenomenon that occurs even in the noninteracting system (unlike superconductivity). Thus, for a weakly interacting gas, it turns out that it is, so to speak, not a self-consistency problem to determine the number of condensed atoms $n_{0}$. For not too low temperatures, the ideal gas formula is quite sufficient, while for $T=0$, the ground state depletion is known to be small. This must also be kept in mind in the discussion of relaxation processes.

We wish to mention, finally, that a situation analogous to the instability of the normal state below $T_{c}$ in superconductors was considered by Levich and Yakhot ${ }^{28}$ for the dilute Bose gas. They couple the Bose gas to a heat bath (like the phonons in metals), whose temperature is suddenly changed from $T>T_{c}$ to $T<T_{c}$. They study the temporal development of the occupation number of the zero-momentum state, which is populated by scattering of particles from other momenta into this state. The characteristic time for this process is found to be given by $\tau_{4}\left(p_{T_{\mathrm{c}}}\right)$, i.e., the normal state 
scattering time (with a slowing down if the final temperature is close to $T_{c}$ ). Clearly, states far from thermal equilibrium are important in this process.

In conclusion, we expect the kinetic equations presented in this article to provide an adequate framework to discuss relaxation processes and transport phenomena in a dilute Bose gas.

\section{APPENDIX A. SCATTERING RATE FROM GREEN'S FUNCTION THEORY}

The Green's function theory of a weakly interacting Bose gas has been, of course, the subject of a large number of investigations. As an illustration, we wish to demonstrate how to calculate the lifetime of the single-particle excitations due to three-excitation processes from microscopic theory. Following Hohenberg and Martin, ${ }^{9}$ one defines, in the presence of an external field that couples directly to the field operators, the Green's functions of the condensate,

$$
G_{1 / 2}^{\alpha}(1)=\sqrt{-i}\left\langle\bar{\psi}_{\alpha}(1)\right\rangle
$$

with $\alpha=1,2 ; \bar{\psi}_{1}=\bar{\psi}, \bar{\psi}_{2}=\bar{\psi}^{+} ; 1 \equiv\left(\mathbf{r}_{1}, t_{1}\right)$, for $t_{1}$ between 0 and $-i / T$, where $T$ is the temperature. The linear response of $G_{1 / 2}^{\alpha}$ with respect to the external field, $\eta_{x}^{\alpha}$, is directly related to the Green's function of the excitations:

$$
\delta G_{1 / 2}^{\alpha}(1)=\sqrt{-i} \int d 1^{\prime} G_{1}^{\alpha \beta}\left(1,1^{\prime}\right) \eta_{x}^{\beta}\left(1^{\prime}\right)
$$

and the sum over repeated indices is implied. Thus the Green's function of the excitations is identified as the order parameter response function, or, more precisely, the retarded function as follows from the analytic continuation of (A2).

Consider first the Bogoliubov approximation, in which the self-energy matrix is given by (for a pointlike repulsive potential of strength $v$ )

$$
\Sigma_{\alpha \beta}^{\mathrm{B}}\left(1,1^{\prime}\right)=n_{0} \nu \delta\left(1-1^{\prime}\right)\left[\begin{array}{ll}
2 & 1 \\
1 & 2
\end{array}\right]_{\alpha \beta}
$$

The chemical potential is related to the self-energy such that the spectrum becomes gapless, namely $\mu=n_{0} v$. The Green's function then has the simple form

$$
\hat{G}_{1}(\omega, \mathbf{q})=\left[\omega \hat{\tau}_{3}-\varepsilon_{q} \hat{1}-n_{0} v \hat{\tau}_{1}\right]^{-1}
$$

where $\omega$ and $\mathbf{q}$ are the Fourier conjugate variables to $t_{1}-t_{1}^{\prime}$ and $\mathbf{r}_{1}-\mathbf{r}_{1}^{\prime}$, and $\varepsilon_{q}=\xi_{q}+n_{0} v$, with $\xi_{a}=q^{2} / 2 m$. Here $\hat{\tau}_{1}$ and $\hat{\tau}_{3}$ denote the Pauli matrices. 
Clearly, all elements of $\hat{G}_{1}$ have a common denominator, given by $D(\omega, q)=$ $\omega^{2}-\omega_{q}^{2}$, with $\omega_{q}^{2}=\xi_{q}^{2}+2 \xi_{q} n_{0} \nu$.

Since we are interested in determining the lifetime of the elementary excitations, we have to investigate the self-energy in second order, the Belyaev approximation (as given in explicit form, e.g., in Ref. 9). Ignoring the real parts of the second-order self-energy, we may write

$$
\hat{G}_{1}^{\mathrm{R}}(\omega, \mathbf{q})=\left[\omega \hat{\tau}_{3}-\varepsilon_{q} \hat{1}-n_{0} \nu \hat{\tau}_{1}+i \hat{\Gamma}(\omega, q)\right]^{-1}
$$

with $\hat{\Gamma}=(i / 2)\left(\hat{\Sigma}^{\mathrm{R}}-\hat{\Sigma}^{\mathrm{A}}\right)$. We also define

$$
\hat{\Gamma}=\Gamma_{3} \hat{\tau}_{3}+\Gamma_{1} \hat{\tau}_{1}+\Gamma_{0} \hat{1}
$$

and realize that for small $\Gamma_{i}$ the denominator of the Green's functions has the simple form

$$
D(\omega, q)=\omega^{2}-\omega_{q}^{2}+2 i \omega \Gamma_{\mathrm{eff}}(\omega, q)
$$

where $\Gamma_{\text {eff }}$ is given by

$$
\Gamma_{\mathrm{eff}}(\omega, q)=\Gamma_{3}(\omega, q)+\frac{\varepsilon_{q}}{\omega} \Gamma_{1}(\omega, q)-\frac{n_{0} v}{\omega} \Gamma_{0}(\omega, q)
$$

Clearly, $\Gamma_{\mathrm{eff}}\left(\omega=\omega_{q}, q\right)$ gives the linewidth of the elementary excitations, or half the scattering rate. Thus, finally, we have to evaluate the elements of the self-energy matrix (its imaginary part), and the combination (A8) gives the desired result. To be explicit, we present the self-energy expression: ${ }^{9}$

$$
\begin{aligned}
\Sigma_{\alpha \beta}\left(1,1^{\prime}\right)= & \sin _{0} \nu^{2}\left[G_{1}^{\alpha \beta}\left(1,1^{\prime}\right) G_{1}^{\gamma \delta}\left(1^{\prime}, 1\right)\right. \\
& +G_{1}^{\alpha \gamma}\left(1,1^{\prime}\right) G_{1}^{\gamma \delta}\left(1^{\prime}, 1\right)+G_{1}^{\gamma \delta}\left(1^{\prime}, 1\right) G_{1}^{\delta \beta}\left(1,1^{\prime}\right) \\
& \left.+G_{1}^{\alpha \gamma}\left(1,1^{\prime}\right) G_{1}^{\delta \beta}\left(1,1^{\prime}\right)+\frac{1}{2} G_{1}^{\gamma \delta}\left(1,1^{\prime}\right) G_{1}^{\delta \gamma}\left(1^{\prime}, 1\right)\right]
\end{aligned}
$$

Here we use the Green's functions as calculated in first order. We perform the analytic continuation with the help of " $>$ " and " $<$ " functions, and use results like

$$
\hat{G}_{1}^{<}(\omega, \mathbf{q})=\hat{A}(\omega, \mathbf{q}) \cdot b_{\omega} ; \quad \hat{G}_{1}^{>}(\omega, \mathbf{q})=\hat{A}(\omega, \mathbf{q}) \cdot\left(1+b_{\omega}\right)
$$

with $b_{\omega}=[\exp (\omega / T)-1]^{-1}$, and $\hat{A}=\hat{G}_{1}^{\mathrm{R}}-\hat{G}_{1}^{\mathrm{A}}$ turns out to be given by

$$
\frac{i \hat{A}(\omega, \mathbf{q})}{2 \pi}=\left[\begin{array}{cc}
u_{q}^{2} & -u_{q} v_{q} \\
-u_{q} v_{q} & v_{q}^{2}
\end{array}\right] \delta\left(\omega-\omega_{q}\right)-\left[\begin{array}{cc}
v_{q}^{2} & -u_{q} v_{q} \\
-u_{q} v_{q} & u_{q}^{2}
\end{array}\right] \delta\left(\omega+\omega_{q}\right)
$$

with the amplitudes $u_{q}$ and $v_{q}$ given by

$$
u_{q}^{2}\left(v_{q}^{2}\right)=\frac{1}{2}\left[\varepsilon_{q} / \omega_{q(-)}^{+} 1\right]
$$


and $u_{q} v_{q}=n_{0} v / 2 \omega_{q}$. The final result can be brought into a rather simple form, namely

$$
\begin{aligned}
\Gamma_{\mathrm{eff}}(\omega, q)= & 2 \pi n_{0} v^{2} \sum_{p_{2}, p_{3}}\left\{W(1 ; 2,3)\left[1+b_{2}+b_{3}\right] \delta(1-2-3)\right. \\
& \left.+2 W(1,2 ; 3)\left[b_{2}-b_{3}\right] \delta(1+2-3)\right\}
\end{aligned}
$$

with $1 \equiv(\omega, \mathbf{q})$,

$$
\delta(1-2-3)=(2 \pi)^{3} \delta\left(\mathbf{q}-\mathbf{p}_{2}-\mathbf{p}_{3}\right) \delta\left(\omega-E_{2}-E_{3}\right)
$$

and $b_{2}=b\left(E_{2}\right)$, etc. Clearly, we identify the two terms as in Eq. (39), describing the decay (the scattering) of a fluctuation with frequency $\omega$ and wave vector $\mathbf{q}$ into (at) elementary excitations, respectively. The W's are given by

$$
\begin{aligned}
& 2 W(1 ; 2,3)=a+\frac{\varepsilon_{q}}{\omega} b+\frac{2 n_{0} v}{\omega} c \\
& 2 W(1,2 ; 3)=\bar{a}+\frac{\varepsilon_{q}}{\omega} \bar{b}+\frac{2 n_{0} v}{\omega} \bar{c}
\end{aligned}
$$

with $a=x^{2}-y^{2}, b=x^{2}+y^{2}, c=x y$, and similarly for $\bar{a}, \bar{b}, \bar{c}$, where

$$
x=u_{2} u_{3}-u_{2} v_{3}-v_{2} u_{3} ; \quad y=v_{2} u_{3}+u_{2} v_{3}-v_{2} v_{3}
$$

and

$$
\bar{x}=u_{2} u_{3}-v_{2} u_{3}+v_{2} v_{3} ; \quad \bar{y}=u_{2} v_{3}-u_{2} u_{3}-v_{2} v_{3}
$$

for $\omega=\omega_{q}$, we realize that

$$
\varepsilon_{q} / \omega \rightarrow u_{1}^{2}+v_{1}^{2} ; \quad n_{0} v / \omega \rightarrow 2 u_{1} v_{1}
$$

and find $W(1 ; 2,3)=2|A(1 ; 2,3)|^{2}$ and $W(1,2 ; 3)=2|A(3 ; 1,2)|^{2}$, with the amplitude $A$ as given in Eq. (41). We thus have confirmed the expression for the scattering rate used in Section 3 [and obtained by linearizing Eq. (39)]. We emphasize that in deriving the expression for $\Gamma_{\text {eff }}$, we did not need to use a particular approximation for the spectrum: With $\varepsilon_{q} \rightarrow$ $\xi_{q}+\sigma_{11}-\phi$ and $n_{0} v \rightarrow \sigma_{12}$, the result is still of the form (A11), (A12).

\section{APPENDIX B. RESPONSE IN THE COLLISIONLESS REGIME (CONSERVING APPROXIMATION)}

In this Appendix we describe in more detail the order parameter response in the collisionless regime within the self-consistent mean field approximation. We consider spatially homogeneous situations only. As in Appendix $\mathrm{A}$, we start from the equations of motion for $\mathbf{G}_{1 / 2}(t)=$ 
$(-i)^{1 / 2} \boldsymbol{\psi}(t)$, where $\psi=\left(\psi, \psi^{*}\right)$ and $\hat{G}_{1}\left(\mathbf{p}, t, t^{\prime}\right)$ :

$$
\left[i \hat{\tau}_{3} \partial_{t}+\mu\right] \boldsymbol{\psi}=\boldsymbol{\eta}+\boldsymbol{\eta}_{x}
$$

where $\boldsymbol{\eta}_{x}=\left(\eta_{x}, \eta_{x}^{*}\right)$ is the external field, and

$$
\left[i \hat{\tau}_{3} \partial_{t}-\left(\frac{p^{2}}{2 m}-\mu\right) \hat{1}-\hat{\Sigma}\right] \hat{G}_{1}=\hat{1}
$$

As discussed briefly in Section 2, it will be convenient to introduce the equal-time " $<$ " Green's function:

$$
\hat{n}_{p}=\hat{n}(\mathbf{p}, t)=i \hat{G}_{1}^{<}(\mathbf{p}, t, t)
$$

The expressions for $\hat{\Sigma}$ and $\boldsymbol{\eta}$ in mean field approximation can be found in Ref. 9; we write

$$
\hat{\Sigma}\left(t, t^{\prime}\right)=\hat{\sigma}(t) \delta\left(t-t^{\prime}\right) ; \quad \eta=\left(\eta_{1}, \eta_{2}\right)
$$

and obtain

$$
\begin{aligned}
\sigma_{11} & =\sigma_{22}=2 v \rho \\
\sigma_{12} & =v\left(\psi^{2}+\sum_{p} \hat{n}_{12}\right) \\
\eta_{1} & =v\left[\psi\left(2 \rho-n_{0}\right)+\psi^{*} \sum_{p} \hat{n}_{12}\right]
\end{aligned}
$$

etc., where $\rho$, the total density, is given by

$$
\rho=n_{0}+\sum_{p} \hat{n}_{11}
$$

As an illustration, consider the equations in equilibrium, in which case $\psi$ is real and equal to $n_{0}^{1 / 2}$. Then we find from (B1) and (B5) that

$$
\mu=\frac{1}{2 n_{0}^{1 / 2}}\left(\eta_{1}+\eta_{2}\right)=\sigma_{11}+\sigma_{12}-2 n_{0} v
$$

which is the analog of Eq. (28).

Finally, we introduce $\hat{\bar{n}}_{p}=\hat{\tau}_{3} \hat{n}_{p}$; it is clear from (B2) that the equation of motion for $\hat{\bar{n}}_{p}$ is obtained by subtracting the "adjoint equation" $\hat{G}_{1} \hat{G}_{1}^{-1}=$ $\hat{1}$. It is of the form of a matrix transport equation:

$$
\partial_{t} \hat{\bar{n}}_{p}+i\left[\hat{\varepsilon}_{p}, \hat{n}_{p}\right]=0
$$

with

$$
\hat{\varepsilon}_{p}=\left[\left(\frac{p^{2}}{2 m}-\mu\right) \hat{1}+\hat{\sigma}\right] \hat{\tau}_{3}
$$


As a direct consequence of these equations, we find the continuity equation in the form

$$
\partial_{t} \rho=i\left(\psi \eta_{x}^{*}-\psi^{*} \eta_{x}\right)=2 n_{0}^{1 / 2} \eta_{x}^{\mathrm{T}}
$$

a result that was mentioned earlier [Eq. (35)].

Of course, introducing a matrix distribution function is of no particular advantage if we are just concerned with the linear response in mean field approximation. We mention, however, that a derivation of the scalar Boltzmann equation can be given by starting from (B8), generalized to include spatial gradients and collisions, and performing a Bogoliubov transformation. $^{29}$

We consider now the linear response to the field $\eta_{x}$, and write

$$
\begin{aligned}
& \psi=n_{0}^{1 / 2}+\delta \psi, \quad \delta \psi=\delta \psi^{\mathrm{L}}+i \delta \psi^{\mathrm{T}} \\
& \hat{n}=\hat{b}+\delta \hat{\bar{n}}_{p}
\end{aligned}
$$

etc. Note that the matrix distribution function in equilibrium $\hat{b}$ is given by

$$
\hat{b}=\frac{1}{2}\left(\frac{\hat{\varepsilon}_{p}}{E_{p}} \operatorname{cth} \frac{E_{p}}{2 T}-\hat{1}\right)
$$

where $E_{p}=\left(\varepsilon_{p}^{2}-\sigma_{12}^{2}\right)^{1 / 2}, \varepsilon_{p}=\xi_{p}-\mu+\sigma_{11}$, is the energy of the singleparticle excitations in the present approximation. In addition, $\delta \psi^{\mathrm{L}, \mathrm{T}}$ are related to $\delta n_{0}$ and $\delta \phi$ (as defined in Section 2) by

$$
\delta n_{0}=2 n_{0}^{1 / 2} \delta \psi^{\mathrm{L}} ; \quad n_{0}^{1 / 2} \delta \phi=i \omega \delta \psi^{\mathrm{T}}
$$

The final result can, of course, be brought into a matrix form:

$$
\mathscr{D}\left(\begin{array}{c}
\delta \psi^{\mathrm{T}} \\
\delta \psi^{\mathrm{L}}
\end{array}\right)=\left(\begin{array}{c}
X_{1} \\
X_{2}
\end{array}\right)
$$

and in $\mathscr{D}$ and $X_{1,2}$ we encounter several integrals involving the equilibrium distribution function, e.g., expressions like $\beta\left[\varepsilon_{p}^{2}\right]$, where

$$
\beta[\cdot]=v \sum_{p} \frac{\operatorname{cth}\left(E_{p} / 2 T\right)}{E_{p}} \frac{1}{4 E_{p}^{2}-(\omega+i 0)^{2}}[\cdot]
$$

Corrections of this type are very small; if we ignore them completely, we find the simple result

$$
\mathscr{D} \approx\left(\begin{array}{cc}
0 & -i \omega \\
i \omega & 2 n_{0} v
\end{array}\right), \quad X_{1} \approx \eta_{x}^{\mathrm{T}}, \quad X_{2} \approx \frac{4 n_{0} v}{-i \omega} \eta_{x}^{\mathrm{T}}+\eta_{x}^{\mathrm{L}}
$$

Note that det $\mathscr{D}=-\omega^{2}$, and the corrections do not modify this behavior.

Consider, as an example, the response to a steplike external field in this approximation. As a response to a longitudinal field $\eta_{x}^{\mathrm{L}}$ we obtain 
$\delta \psi^{\mathrm{L}}=0$ and $i \omega \delta \psi^{\mathrm{T}}=\delta \eta_{x}^{\mathrm{L}}$, i.e., the number of condensed atoms $n_{0}$ remains unchanged, and the phase of the order parameter is going to rotate. In response to a transverse field $\eta_{x}^{\mathrm{T}}$, on the other hand, we obtain an increase of the total density linearly in time, and the additional particles are simply added to the condensate. In addition, the phase is accelerated according to $\omega^{2} \delta \psi^{\mathrm{T}}=2 n_{0} v \delta \eta_{x}^{\mathrm{T}}$.

As an illustration, we consider now the effect of the small corrections in a situation where $\eta_{x}^{\mathrm{T}}=0$. Expanding (B14) with respect to $\beta[\cdot]$, we find

$$
\begin{aligned}
\delta \psi^{\mathrm{L}} & \approx-n_{0} v \beta[1] \eta_{\dot{x}}^{\mathrm{L}} \\
-i \omega \delta \psi^{\mathrm{T}}-\delta \eta_{x}^{\mathrm{L}} & \approx 2 n_{0} v \beta\left[\xi_{p}\right] \delta \eta_{x}^{\mathrm{L}}
\end{aligned}
$$

The corrections thus modify the rotation frequency of the phase and give a small change in $n_{0}$. For $\eta_{x}^{\mathrm{L}}=\theta(t)$ we find from (B17) that

$$
\delta \psi^{\mathrm{L}}(t)=-\theta(t) n_{0} v^{2} \sum_{p} \frac{\mathrm{cth}\left(E_{p} / 2 T\right)}{4 E_{p}^{3}}\left[1-\cos \left(2 E_{p} t\right)\right]
$$

Clearly, this result is only well defined if the excitation spectrum has a gap. For example, we obtain

$$
\delta \psi^{\mathrm{L}}(\infty)=-\frac{n_{0} v^{2} T}{16 \pi c^{3} \Delta}, \quad m c^{2} \approx n_{0} v
$$

for not too low temperatures $(T \gg \Delta)$. For short times, say $\Delta \ll t^{-1} \ll T, n_{0} v$, the behavior is easily calculated, since in this regime a linear spectrum can be used. With $y(t)=\delta \psi^{\mathrm{L}}(t) / \delta \psi^{\mathrm{L}}(\infty)$, one finds $y(t) \approx 4 \Delta \cdot t$, an increase linear in time. On the other hand, for long times, $t^{-1} \ll \Delta$,

$$
y(t)-y(\infty) \approx-\frac{4}{(2 \pi)^{1 / 2}}(2 \Delta t)^{-3 / 2} \cos \left(2 \Delta t+\frac{\pi}{4}\right)
$$

i.e., $y(t)$ oscillates with a frequency $2 \Delta$ and approaches algebraically its limiting value.* Similar conclusions can be reached for the correction to the simple behavior as given by Eq. (B18). Unfortunately, these "interesting" effects should be considered part of the unphysical consequences a certain approximation can have: Recall that the magnitude of the gap depends on the order of the approximation.

In conclusion, we remark that Eq. (B14) in the low-frequency limit can also be derived from the kinetic theory provided we ignore the collision processes. Essentially, we arrive at the same equations, except that expressions like (B15) are evaluated at zero frequency, and the kinetic equations thus ignore oscillation as given in (B21). In addition, the small corrections of the type $\beta[\cdot]$ are related to $K_{1}$ and $K_{2}$ in Eqs. (59) and *A similar behavior is found in superconductors (see Ref. 27), where the gap has physical significance. 
(60), i.e., to variations of the coherence factors; we noted already that these are small.

\section{ACKNOWLEDGMENTS}

This article was initiated by stimulating discussions with Andrei Ruckenstein and Eric Siggia on spin-polarized hydrogen. I am especially grateful to Fritz Haake for his collaboration on parts of this work, which started during our stay at Cornell University. Interesting discussions with Reiner Kree and Albert Schmid are also acknowledged.

\section{REFERENCES}

1. I. F. Silvera and J. T. M. Walraven, Phys. Rev. Lett. 44, 164 (1980).

2. W. J. Mullin, Phys. Rev. Lett. 44, 1420 (1980).

3. E. D. Siggia and A. E. Ruckenstein, Phys. Rev. Lett. 44, 1423 (1980); J. Phys. (Paris) 41, C7-15 (1980).

4. E. D. Siggia and A. E. Ruckenstein, Phys. Rev. B 23, 3580 (1981).

5. I. F. Silvera, Physica 109+110B, 1499 (1982).

6. N. N. Bogoliubov, J. Phys. (USSR) 11, 23 (1947).

7. S. T. Belyaev, Zh. Eksp. Teor. Fiz. 34, 417, 433 (1958) [Sov. Phys.-JETP 7, 289, 299 (1958)].

8. P. C. Martin, I. Math Phys. 4, 208 (1963).

9. P. C. Hohenberg and P. C. Martin, Ann. Phys. (N. Y.) 34, 291 (1965).

10. N. M. Hugenholtz and D. Pines, Phys. Rev. 116, 489 (1959).

11. Yu. A. Nepomnyashchii and A. A. Nepomnyashchii, Zh. Eksp. Teor. Fiz. 75, 976 (1978) [Sov. Phys.-JETP 48, 493 (1978)].

12. V. N. Popov and A. V. Seredniakov, Zh. Eksp. Teor. Fiz. 77, 377 (1979) [Sov. Phys.-JETP 50, $193(1979)]$.

13. M. Girardeau and R. Arnowitt, Phys. Rev. 113, 755 (1959).

14. I. M. Khalatnikov, in The Physics of Liquid and Solid Helium, K. H. Bennemann and J. B. Ketterson, eds. (Wiley, New York, 1976), Vol. 1, p. 1.

15. A. G. Aronov and V. L. Gurevich, Fiz. Tverd. Tela 16, 2656 (1974) [Sov. Phys.-Solid State 16, $1722(1975)]$.

16. A. A. Abrikosov, L. P. Gorkov, and I. E. Dzyaloshinski, Methods of Quantum Field Theory in Statistical Physics (Dover, New York, 1975).

17. H. Gould and V. K. Wong, Phys. Rev. A 4, 719 (1971).

18. V. L. Ginzburg, Fiz. Tverd. Tela 2, 2031 (1960) [Sov. Phys.-Solid State 2, $1824(1960)$ ].

19. E. P. Gross, Nuovo Cimento 20, 454 (1961); L. P. Pitaevski, Zh. Eksp. Teor. Fiz. 40, 646 (1961) [Sov. Phys.-JETP 13, 451 (1961)].

20. D. G. Friend and R. D. Etters, J. Low Temp. Phys. 39, 409 (1980).

21. U. Eckern and F. Haake, Physica 109+110B, 2114 (1982).

22. F. Mohling and S. Morita, Phys. Rev. 120, 681 (1960).

23. A. Schmid and G. Schön, J. Low Temp. Phys. 20, 207 (1975).

24. D. N. Langenberg, in Proceedings of the 14th International Conference on Low Temperature Physics, M. Krusius and M. Vuorio, eds. (North-Holland, Amsterdam, 1975), Vol. 5 , p. 223.

25. A. Schmid, J. Phys. (Paris) 39, C6-1360 (1978).

26. D. C. Mattis and J. Bardeen, Phys. Rev. 111, 412 (1958).

27. Yu. N. Ovchinnikov, Zh. Eksp. Teor. Fiz. 59, 128 (1970) [Sov. Phys.-JETP 32, 72 (1971)]; Yu. N. Ovchinnikov and G. Schön, preprint.

28. E. Levich and V. Yakhot, Phys. Rev. B 15, 243 (1977); J. Low Temp. Phys. 27, 107 (1977),

29. O. Betbeder-Matibet and P. Nozières, Ann. Phys. (N.Y.) 51, 392 (1969). 